\title{
X-RAY POWER DENSITY SPECTRUM OF THE NARROW-LINE SEYFERT 1 GALAXY ARAKELIAN 564

\author{
Ken Pounds, ${ }^{1}$ Rick Edelson, ${ }^{1,2}$ Alex Markowitz, ${ }^{2}$ and Simon Vaughan ${ }^{1,3}$ \\ Received 2000 December 12; accepted 2001 January 31; published 2001 March 12
}

\begin{abstract}
Beginning in 1999 January, the bright, strongly variable narrow-line Seyfert 1 (NLS1) galaxy Akn 564 has been observed by RXTE once every $\sim 4.3$ days. It was also monitored every $~ 3.2 \mathrm{hr}$ throughout $2000 \mathrm{July}$. These evenly sampled observations have allowed the first quantitative comparison of long- and short-timescale X-ray variability in an NLS1 galaxy and the derivation of an X-ray power density spectrum (PDS). The variability amplitude in the short-timescale light curve is very similar to that in the long-timescale light curve, in marked contrast to the stronger variability on longer timescales, which is characteristic of "normal" broad-line Seyfert 1 (BLS1) galaxies. Furthermore, the Akn 564 PDS power law cuts off at a frequency of $8.7 \times 10^{-7} \mathrm{~Hz}$, corresponding to a timescale of $\sim 13$ days, significantly shorter than that seen in the PDS of NGC 3516, a BLS1 galaxy of comparable luminosity. This result is consistent with NLS1 galaxies showing faster (as opposed to larger amplitude) variations than BLS1 galaxies, providing further evidence that NLS1 galaxies harbor lower mass black holes than BLS1 galaxies of similar luminosity, accreting at a correspondingly higher relative rate.

Subject headings: galaxies: active — galaxies: individual (Arakelian 564) — galaxies: Seyfert — X-rays: galaxies
\end{abstract}

\section{INTRODUCTION}

A relatively recent development in the taxonomy of active galactic nuclei (AGNs) has been the emergence of narrow-line Seyfert 1 (NLS1) galaxies as an important subclass. NLS1 galaxies were originally identified on the basis of their optical properties, exhibiting narrower permitted lines $(\mathrm{H} \beta$ FWHM $<2000 \mathrm{~km} \mathrm{~s}^{-1}$ ) than "normal" broad-line Seyfert 1 (BLS1) galaxies and weaker $[\mathrm{O}$ III] $/ \mathrm{H} \beta<3$ than Seyfert 2 galaxies (Osterbrock \& Pogge 1985; Goodrich 1989). ROSAT data showed these properties to be strongly correlated with the Xray spectral slope, in the sense that NLS1 galaxies tend to have steep soft X-ray spectra (Boller, Brandt, \& Fink 1996). In addition, many NLS1 galaxies are strongly variable on timescales of hours or less, with some NLS1 galaxies showing giant (factor of 100) X-ray flares over timescales of days (e.g., Brandt et al. 1999). On these short timescales the variability levels in NLS1 galaxies are typically a factor of 3-10 times larger than those seen in BLS1 galaxies of similar luminosity (Turner et al. 1999; Leighly 1999).

A number of models have been proposed to explain the distinctive properties observed in NLS1 galaxies. The most widely accepted explanation is that NLS1 galaxies are accreting near the Eddington limit (Pounds, Done, \& Osborne 1995; Laor et al. 1997), while BLS1 galaxies accrete at a lower rate. On this interpretation, the high accretion rate is directly responsible for the strong soft X-ray emission, by enhanced thermal radiation from the accretion disk, while the increased soft photon flux cools the hard X-ray source leading to the steeper power law frequently seen at higher X-ray energies in NLS1 galaxies (Brandt, Mathur, \& Elvis 1997). It is the prospect that comparative study of NLS1 and BLS1 galaxies will shed light on the accretion rate, one of the fundamental parameters of an AGN, that makes the emergence of the new subclass of AGNs of such interest and importance.

\footnotetext{
${ }^{1}$ X-ray Astronomy Group, University of Leicester, Leicester LE1 7RH, England, UK; kap@star.le.ac.uk

${ }^{2}$ Astronomy Department, University of California at Los Angeles, Los Angeles, CA 90095-1562.

${ }^{3}$ Institute of Astronomy, University of Cambridge, Madingley Road, Cambridge CB3 OHA, England, UK.
}

$\mathrm{X}$-ray variability has been recognized as a powerful probe of the central regions of AGNs since the EXOSAT "long-looks" first showed (McHardy 1988) that rapid variability was common in Seyfert galaxies, lending early support to the nowstandard black hole/accretion disk paradigm. The EXOSAT data were found to be well described by a fluctuation power density spectrum (PDS) rising smoothly to lower frequencies as a power law, $f^{-\alpha}$, where $\alpha=1-2$, to a limit imposed by the maximum duration of the $\sim 2$ day observations (e.g., Lawrence \& Papadakis 1993). Attempts to constrain a flattening or "cutoff" in the PDS (as required to avoid the variability power diverging) were made by combining EXOSAT data with earlier X-ray satellite observations of NGC 5506 (McHardy 1988) and NGC 4151 (Papadakis \& McHardy 1995). These analyses yielded evidence for a cutoff timescale of several weeks; however, the uneven sampling of these data made their reliability uncertain. The observational situation was significantly improved with the launch of the Rossi X-Ray Timing Explorer $(R X T E)$. Taking advantage of the unique properties of this satellite, Edelson \& Nandra (1999) obtained evenly sampled fluxes of the BLS1 galaxy NGC 3516 on long, medium, and short timescales and combined these data to produce a PDS covering four decades in temporal frequency, finding a cutoff timescale of $\sim 1$ month.

Until now the only quantitative assessment of the rapid X-ray variability of NLS1 galaxies has been based on measurements of the excess variance, obtained from ASCA observations, typically of 1 day duration. Based on these data, Turner et al. (1999) and Leighly (1999) found the short-term variability of NLS1 galaxies to be substantially greater than for BLS1 galaxies, while in both cases the excess variance was anticorrelated with the Xray luminosity. The present Letter reports the results of the first extensive and evenly sampled X-ray monitoring of an NLS1 galaxy, using RXTE to monitor Akn 564 over a period of nearly 2 yr. Akn 564 is particularly well suited for this study, being the brightest known NLS1 galaxy in the hard X-ray sky $[F(2-$ $10 \mathrm{keV}) \approx(2-5) \times 10^{-11} \mathrm{ergs} \mathrm{cm}^{-2} \mathrm{~s}^{-1}$; Vaughan et al. 1999a], strongly variable (e.g., $50 \%$ variations on timescales of hours; Leighly 1999), and located well out of the ecliptic plane (allowing monitoring by $R X T E$ throughout the year). Subsequent to 
TABLE 1

SAMPLing Parameters

\begin{tabular}{cccccc}
\hline \hline Timescale & $\begin{array}{c}\text { Observing Dates } \\
\text { (MJD) }\end{array}$ & Mean Sampling Interval & $\begin{array}{c}\text { Data Lost } \\
\text { (points) }\end{array}$ & Number of Flux Points & $\begin{array}{c}\text { Usable Temporal } \\
\text { Frequency Range }\end{array}$ \\
\hline Long $\ldots . .$. & $51,179.55-51,806.78$ & 4.267 days & 9 & 148 & $6.9 \times 10^{-8}-1.1 \times 10^{-6}$ \\
Short $\ldots . .$. & $51,694.82-51,726.47$ & $3.2 \mathrm{hr}$ & 13 & 235 & $1.4 \times 10^{-6}-3.4 \times 10^{-5}$ \\
\hline
\end{tabular}

the start of our RXTE monitoring campaign, Akn 564 was chosen for simultaneous monitoring with the Hubble Space Telescope, $A S C A$, Chandra, and the AGN Watch network of ground-based optical telescopes.

\section{OBSERVATIONS AND DATA REDUCTION}

Our X-ray monitoring was planned to obtain variability information spanning timescales from hours to months. Akn 564 was observed once every 4.3 days (=64 orbits) from 1999 January 1 to 2000 September 19 (the limit of the present analysis, although this low-frequency monitoring is continuing) and once every $\sim 3.2 \mathrm{hr}$ (=2 orbits) from 2000 June 30 to August 1. (Twice daily observations were also obtained from 1999 October 25 to November 11, in support of the AGN Watch campaign; O. Schemmer et al. 2001, in preparation). Our sampling parameters are summarized in Table 1.

The RXTE Proportional Counter Array (PCA) consists of five collimated proportional counter units (PCUs), sensitive in a nominal 2-60 keV bandpass (Jahoda et al. 1996). At the start of the present campaign, three of the PCUs $(0,1$, and 2$)$ were in routine

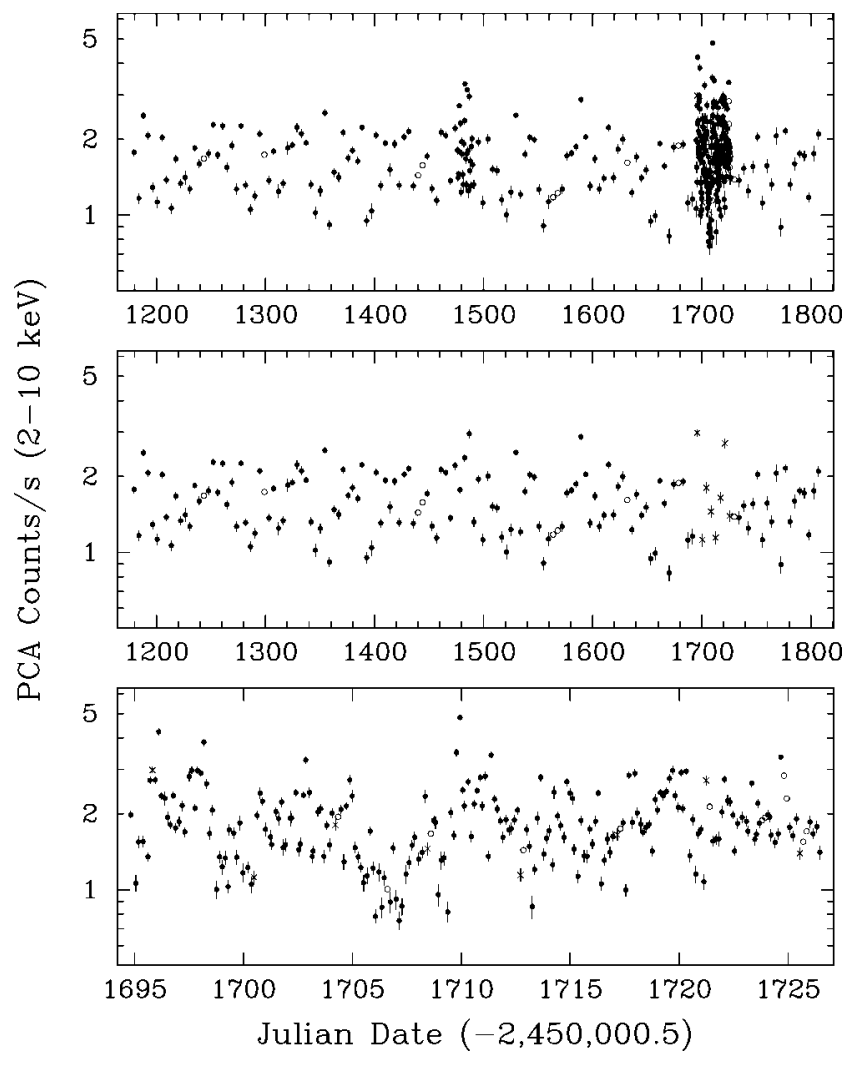

FIG. 1.-Full-band (2-10 keV) light curve (top), resampled on long (4.3 days to 20 months; middle) and short ( $3.2 \mathrm{hr}$ to 31 days; bottom) timescales. These center and bottom light curves were produced by filtering and interpolating the data in the top panel as described in the text. Interpolated points are shown as open circles without error bars, and the eight points common to both light curves as crosses. use. After the gain settings of all five PCUs on board RXTE were changed on 1999 March 22, this number was reduced to two (0 and 2) and then, after 2000 May 12, to one (2). Data collected during each of the two gain epochs were extracted separately using background model files appropriate to that gain epoch. The present analysis is restricted to the $2-10 \mathrm{keV}$ band, where the PCA is most sensitive and the systematic errors are best understood. Data from the top (most sensitive) layer of the PCU array were extracted using the REX reduction script, ${ }^{4}$ and background counts were estimated using the "L7-240" model. Background-subtracted count rates were then obtained in each epoch to assign a mean flux and error. Count rates derived from data taken during the earlier gain setting were scaled to account for the effect of the gain change by a factor derived from observations of Cassiopeia A and then normalized to units of counts per second per PCU. Figure 1 (top panel) reproduces the full light curve.

\section{TEMPORAL ANALYSIS}

Before performing the time series analyses described below, the observed light curve was resampled to provide two nearly independent light curves, one sampling short timescales ( days) and the other, long timescales ( months). In Figure 1, the middle panel contains the full 20 months of data, sampled on a grid as close to 4.3 days as possible. Interpolating over the nine data points that were missing during this period and mapping to an even grid yielded a total of 148 evenly spaced flux points. This had the effect of filtering out (not smoothing) most of the data in the intensive periods around MJD 51,480 and 51,700. The short-timescale light curve, shown in the bottom panel of Figure 1, was obtained during the 32 day intensive sampling period from 2000 June 30 to August 1. Interpolating over the 13 data points missing during this period yielded a total of 235 evenly spaced flux points.

\subsection{Comparison of Long- and Short-Timescale Light Curves}

The long- and short-timescale data are quantitatively compared in Table 2, where column (2) gives the mean count rates and column (3) the fractional excess variance, $\sigma_{\mathrm{Xs}}^{2}$, defined as

$$
\sigma_{\mathrm{XS}}^{2}=\frac{S^{2}-\left\langle\sigma_{\mathrm{err}}^{2}\right\rangle}{\langle X\rangle^{2}}
$$

\begin{tabular}{|c|c|c|c|}
\hline \multicolumn{4}{|c|}{$\begin{array}{l}\text { See http://heasarc.gsfc.nasa.gov/docs/xte/ } \\
\text { See http://heawww.gsfc.nasa.gov/ keith/ } \\
\text { TABLE } 2 \\
\text { MuLTIBAND LIGHT CURV }\end{array}$} \\
\hline $\begin{array}{l}\text { Timescale } \\
\text { (1) }\end{array}$ & $\begin{array}{c}\text { Mean } \\
\left(\text { counts } s^{-1}\right) \\
\text { (2) }\end{array}$ & $\begin{array}{c}\text { Excess Variance } \\
\text { (3) }\end{array}$ & $\begin{array}{c}\text { Power-Law Slope } \\
\text { (4) }\end{array}$ \\
\hline Long..... & $1.63 \pm 0.04$ & $0.076 \pm 0.009$ & $-0.24 \pm 0.08$ \\
\hline Short . & $1.87 \pm 0.04$ & $0.111 \pm 0.010$ & $-0.96 \pm 0.07$ \\
\hline
\end{tabular}




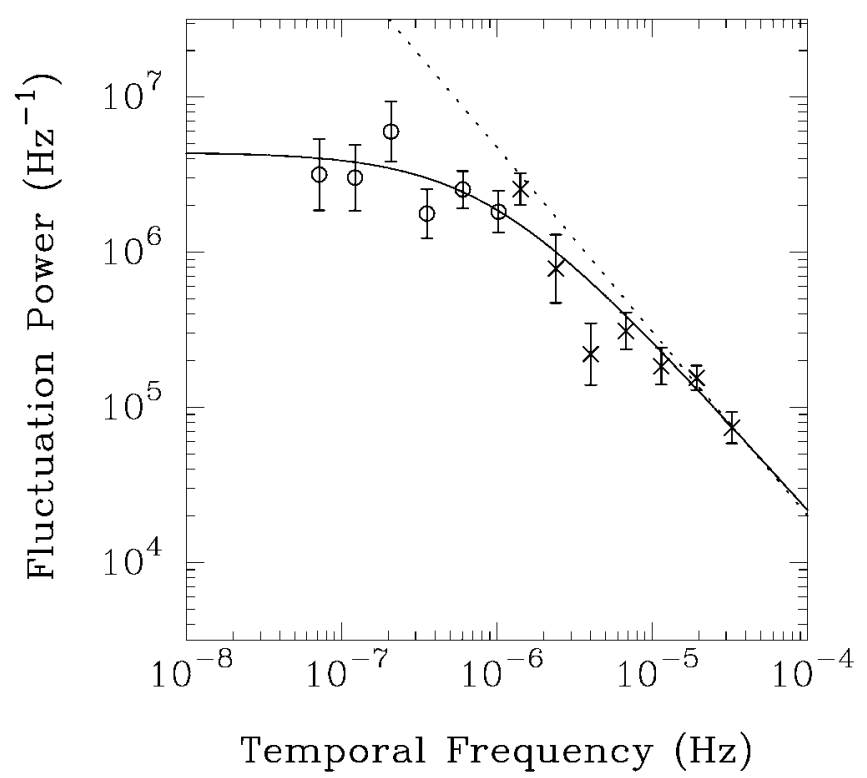

FIG. 2.-Combined PDS of Akn 564. The crosses refer to the PDS derived from the short-term light curve, while the circles are from the long-timescale PDS. The solid line is the best fit as described in the text, a power law with a low-frequency cutoff at $\sim 13$ days. The dotted line is the same power law without the cutoff.

where $\langle X\rangle$ is the mean flux, $\left\langle\sigma_{\text {err }}^{2}\right\rangle$ is the mean square error, and $S^{2}$ is the measured variance of the light curve (e.g., Nandra et al. 1997). We have estimated $( \pm 1 \sigma)$ errors on the excess variance as $\pm \sigma_{\mathrm{xS}}^{2}(2 / N)^{1 / 2}$, where $N$ is the number of flux points; note that for Gaussian fluctuations $S^{2}$ will follow a $\chi^{2}$ distribution (R. Warwick 2000, private communication).

The short-timescale light curve of Akn 564 shows a marginally larger excess variance than the long-timescale light curve, a result that is strikingly different from that of BLS1 galaxies. In a survey of RXTE and ASCA data for eight BLS1 galaxies, Markowitz \& Edelson (2001) found larger values of $\sigma_{\mathrm{XS}}^{2}$ on long ( months) than on short ( $\sim$ hours) timescales in every case. This comparison strongly suggests that largeamplitude X-ray variability is more rapid, i.e., extends to significantly shorter timescales, in Akn 564 than in BLS1 galaxies of comparable luminosity.

\subsection{Fluctuation Power Density Spectrum}

In order to better quantify the short-term variability of Akn 564, the PDS was then constructed following the prescription of Edelson \& Nandra (1999), first determining separate shortand long-timescale PDSs and then combining them to produce a single PDS. The individual PDSs were derived using a direct discrete Fourier transform (Oppenheim \& Shafer 1975; Brillinger 1981). The zero-power and next two (very noisy) lowest frequency points of each PDS were ignored and the remaining points binned every factor of 1.7 (0.23 in the logarithm). Powerlaw models were then fitted separately to the long- and shorttimescale PDSs. The slope and uncertainty, measured from an unweighted, least-squares fit to the logarithmically binned data and derived using the Welch window function, are given in column (4) of Table 2 . These show a highly significant $(10 \sigma)$ systematic flattening from short timescales (with $a=$ $-0.96 \pm 0.07)$ to long timescales $(a=-0.24 \pm 0.08)$.

The long- and short-timescale PDSs were then combined. Unlike Edelson \& Nandra (1999), who allowed the relative
TABLE 3

PDS Fit PaRameters

\begin{tabular}{ccc}
\hline \hline Description & Parameter & Value \\
\hline High-frequency slope $\ldots \ldots \ldots \ldots$ & $a$ & $-1.12 \pm 0.04$ \\
Cutoff frequency $\ldots \ldots \ldots \ldots \ldots$. & $f_{c}$ & $(8.72 \pm 0.81) \times 10^{-7} \mathrm{~Hz}$ \\
Cutoff timescale $\ldots \ldots \ldots \ldots \ldots$ & $t_{c}\left(=1 / f_{c}\right)$ & $13.3_{-1.2}^{+1.3} \mathrm{days}$ \\
Normalization coefficient $\ldots \ldots$. & $C_{1}$ & $(4.4 \pm 0.2) \times 10^{6} \mathrm{~Hz}^{-1}$ \\
\hline
\end{tabular}

normalization between PDSs to float as a free parameter, we retained the absolute normalizations. The combined PDS is shown in Figure 2. A simple power law gave an unacceptable fit $(>99.99 \%)$, with a reduced $\chi^{2}$ of $\chi_{v}^{2}=3.06$ for 13 degrees of freedom.

The combined PDS was then fitted with a model in which a steep power law dominates at high frequencies but cuts off to a slope of $a=0$ at low frequencies:

$$
P(f)=C /\left(1+f / f_{c}\right)^{a},
$$

where $P(f)$ is the fluctuation power at a frequency $f, a$ is the power-law slope at high temporal frequencies, $f_{c}$ is the "cutoff frequency," well below which the PDS flattens to a slope of zero, and $C$ is the normalization. The fit, with three free parameters $\left(C, f_{c}\right.$, and $\left.a\right)$ was a significant improvement over the simple power law, with $\chi_{v}^{2}=1.70$ for 12 degrees of freedom, although still formally unacceptable ( $94 \%$ confidence) mainly because of the higher absolute level of variability power in the short-timescale data. The high-frequency slope of the broken power-law fit is $a=-1.12$, similar to the slope derived using the high-frequency PDS alone, and the cutoff frequency of $8.7 \times 10^{-7} \mathrm{~Hz}$ corresponds to a timescale of $\sim 13$ days. These parameters are summarized in Table 3 . While the power law plus cutoff model is clearly a marked improvement over the unbroken power law, we note that the exact shape of the turnover is not well constrained and the low-frequency behavior not at all well determined.

\section{DISCUSSION AND CONCLUSIONS}

We have carried out the most comprehensive $\mathrm{X}$-ray variability study of an NLS1 galaxy to date. The long- and short-timescale light curves of Akn 564 are found to be remarkably similar, with comparable excess variances over the sampling intervals of 32 and 627 days. The straightforward deduction from a visual examination of the light curves is that Akn 564 exhibits most of its variability power on timescales substantially less than 1 month. We suggest this may well be a common feature of NLS1 galaxies and one that distinguishes the subclass from BLS1 galaxies. Turner et al. (1999) calculated the excess variance for ASCA observations of 36 Seyfert galaxies over a typical sampling interval of 1 day, finding values up to $\sim 10$ times greater for NLS1 galaxies compared with BLS1 galaxies (of similar luminosity). A current explanation for the rapid and large amplitude variability characteristic of narrow-line AGNs is in terms of a smaller black hole mass and hence of size scale. Our result now confirms-for Akn 564 - that the large short-term variability is indeed primarily due to the timescales being shorter (rather than-necessarily-having greater variability power) than for BLS1 galaxies of comparable luminosity.

The Akn 564 PDS now offers an opportunity to quantify the lower mass and higher accretion rate of an NLS1 galaxy in comparison with a BLS1 galaxy of similar luminosity. To date the only BLS1 galaxy PDS obtained by a similar evenly sam- 
pled campaign is for NGC 3516, from which Edelson \& Nandra (1999) obtained a cutoff timescale of $~ 30$ days. Assuming for the moment that a simple scaling law applies for the variability timescale and black hole mass of NLS1 and BLS1 galaxies, our result suggests that Akn 564 has a black hole mass of order $\sim 0.4$ that of NGC 3516. Reverberation mapping by Robinson (1994) found NGC 3516 to have a black hole mass of order $\sim 3 \times 10^{7} M_{\odot}$, implying a corresponding mass for Akn 564 of $\sim 1 \times 10^{7} M_{\odot}$. Estimation of the accretion rate for most AGNs is hampered by a large uncertainty of the luminosity in the hidden EUV band. However, Wandel, Peterson, \& Malkan (1999) showed the bolometric luminosity of BLS1 galaxies may be estimated by scaling from the continuum luminosity at $5100 \AA$, based on a comparison of photoionization and reverberation estimates of black hole masses. By this means, we derive a bolometric luminosity for NGC 3516 of $\sim 10^{44}$ ergs $\mathrm{s}^{-1}$, which should be good to a factor of $\sim 3$. The corresponding accretion rate for NGC 3516 is then of order $0.05 \dot{M}_{\text {Edd }}$.

The very different spectral energy distributions of NLS1 galaxies means that we cannot rely on the Wandel et al. (1999) relation to estimate the bolometric luminosity of Akn 564. Instead we use the respective $2-10 \mathrm{keV}$ luminosities, for which Akn 564 is some $\sim 3$ times that of NGC 3516. Combining the respective estimates of black hole mass and luminosity then gives an accretion rate some $\sim 8$ times larger for Akn 564 than for NGC 3516, indicating that Akn 564 is accreting at a substantial fraction of the Eddington limit, or $0.4 \dot{M}_{\text {Edd }}$. We note, furthermore, that this is probably a lower limit, given that the scaling with NGC 3516 via the $2-10 \mathrm{keV}$ luminosities takes no account of the strong soft X-ray excess in Akn 564.

Interpreting the PDS in the above way is subject to two caveats. First, is the variability stationary, to at least the extent that the measured "characteristic timescale" is a reliable mea- sure of scale and black hole mass? The uniformity of the longtimescale light curve of Akn 564 shown in Figure 1 is reassuring in that respect, suggesting the absence of low-frequency power is sustained throughout our campaign. On the other hand, the higher mean flux and larger fractional variability during the intensive monitoring suggests a degree of nonstationarity, but not to an extent that alters our basic finding, that Akn 564 contains most of its variability power on timescales much shorter than 1 month. A second question is whether the accretion disks in both Akn 564 and NGC 3516 extend close to the innermost stable orbit, justifying our inverse scaling of black hole mass with the respective values of $f_{c}$. The high luminosity of NLS1 galaxies indicates that is generally true for NLS1 galaxies, while in the case of NGC 3516 the observation of a broad Fe K fluorescence line (Nandra et al. 1999) suggests that its accretion disk also extends close to the innermost stable orbit.

We conclude that comparisons of the X-ray variability of Akn 564 and NGC 3516 indicate a black hole mass for Akn 564 of $\sim 1 \times 10^{7} M_{\odot}$, implying an accretion rate in the range $0.2 \dot{M}_{\mathrm{Edd}}-1.0 \dot{M}_{\mathrm{Edd}}$. It is interesting to compare this result with the accretion rate derived from modeling line widths and variability timescales in the optical broad-line region (Laor 1998). Taking the above estimate for the luminosity of Akn 564 and $\mathrm{H} \beta \mathrm{FWHM}=800 \mathrm{~km} \mathrm{~s}^{-1}$ (Vaughan et al. 1999b), the relation in Laor (1998) yields an "independent" estimate for Akn 564 of $1.0 \dot{M}_{\mathrm{Edd}}$.

The authors acknowledge the dedicated work of the RXTE team, especially Evan Smith for his careful scheduling so close to the ephemeris. R. E. and A. M. were supported by NASA grants NAG5-7315 and NAG5-9023, and S. V. by the UK Particle Physics and Astronomy Research Council.

\section{REFERENCES}

Boller, Th., Brandt, W. N., \& Fink, H. 1996, A\&A, 305, 53

Brandt, W. N., Boller, Th., Fabian, A. C., \& Ruszkowski, M. 1999, MNRAS, 303, L53

Brandt, W. N., Mathur, S., \& Elvis, M. 1997, MNRAS, 285, L25

Brillinger, D. 1981, Time Series: Data Analysis and Theory (2d ed.; San Francisco: Holden-Day)

Edelson, R., \& Nandra, K. 1999, ApJ, 514, 682

Goodrich, R. W. 1989, ApJ, 342, 224

Jahoda, K., Swank, J., Giles, A., Stark, M., Strohmayer, T., Zhang, W., \& Morgan, E. H. 1996, Proc. SPIE, 2808, 59

Laor, A. 1998, ApJ, 505, L83

Laor, A., et al. 1997, ApJ, 477, 93

Lawrence, A., \& Papadakis, I. 1993, ApJ, 414, L85

Leighly, K. 1999, ApJS, 125, 297

Markowitz, A., \& Edelson, R. 2001, ApJ, 547, 684

McHardy, I. 1988, Mem. Soc. Astron. Italiana, 59, 239
Nandra, K., George, I. M., Mushotzky, R. F., Turner, T. J., \& Yaqoob, T. 1997, ApJ, 476, 70

. 1999, ApJ, 523, L17

Oppenheim, A., \& Shafer, R. 1975, Digital Signal Processing (Paramus: Prentice-Hall)

Osterbrock, D. E., \& Pogge, R. W. 1985, ApJ, 297, 166

Papadakis, I., \& McHardy, I. 1995, MNRAS, 273, 923

Pounds, K. A., Done, C., \& Osborne, J. 1995, MNRAS, 277, L5

Robinson, A. 1994, in ASP Conf. Ser. 69, Reverberation Mapping of the BroadLine Region in Active Galactic Nuclei, ed. P. M. Gondhalekar, K. Horne, \& B. M. Peterson (San Francisco: ASP), 147

Turner, T. J., George, I. M., Nandra, K., \& Turcan, D. 1999, ApJ, 524, 667

Vaughan, S., Pounds, K. A., Reeves, J. N., Warwick, R. S., \& Edelson, R. A. 1999a, MNRAS, 308, L34

Vaughan, S., Reeves, J. N., Warwick, R. S., \& Edelson, R. A. 1999b, MNRAS, 309,113

Wandel, A., Peterson, B. M., \& Malkan, M. A. 1999, ApJ, 526, 579 